\title{
From Valsartan to Ranitidine: The Story of Nitrosamines So Far
}

\section{Dear Readers,}

Every point in the process of caregiving contains a certain degree of inherent unsafety!

The very idea of patient safety is the prevention of adverse effects to patients associated with health care. While healthcare has become more effective, it has also become more complex with extensive use of new technologies, medicines and treatments.

The expectations of health-system performance are mounting, challenging its readiness to change and adjust to technological development and emerging health threats.

Nitrosamines are a class of chemical compounds that were first described in the chemical literature over 100 years ago, but not until 1956 did they receive much attention. These are chemical compounds having a nitroso group bonded to an amine. Most nitrosamines are carcinogenic.

In 1956, Magee and Barnes showed the carcinogenic potential of Nitrosodimethylamine (NDMA) in rats. Since then, about $90 \%$ of the 300 tested nitrosamines have shown potent carcinogenic effects in experimental studies in at least 39 different species and many different organs. Predominant sites of tumour induction in animals include oral cavity, oesophagus, stomach, urinary bladder and brain. This landmark discovery caused scientists around the world to investigate the carcinogenic properties of other nitrosamines and N-nitroso compounds.

Nitrosamines occur commonly because their chemical precursor's amines and nitrosating agents react to form nitrosamine, however this fact remains quite facile. Therefore, only two specific $\mathrm{N}$-nitroso compounds, NDMA and NDEA (N-Nitrosodiethylamine), have been classified by the International Agency for Research on Cancer (IARC) as "probably carcinogenic to humans".

This discovery compelled scientists to begin asking serious questions about the occurrence of nitrosamines-could nitrosamines be formed in human foods?

With the invention of reliable analytical methods being developed to determine nitrosamine levels in foods and beverages during 1970s and 1980s, such questions could be answered with more authenticity and hence the question of NDMA being present in medicines that are commonly used came under the scanner of drug makers and regulators.

Contamination with NDMA and related nitrosamine compounds has been a vexing problem for drug makers and the FDA since July 2018, when NDMA was found in certain Chinese-made valsartan products. The recalls later extended to other ARBs including losartan and irbesartan. Certain manufacturing processes were thought to be responsible and the agency has been working with manufacturers to avoid introduction of nitrosamines into these agents.

Sartans such as candesartan, irbesartan, losartan, olmesartan and valsartan, which belong to a class of medicines angiotensinII-receptor antagonists (ARBs) are under the radar for presence of NDMA. These medicines are used to treat patients with hypertension and those with certain heart or kidney diseases.
DOI: 10.5530/ijopp.13.1.1

Address for

correspondence:

Dr. Mahvash Iram,

Assistant Editor, ijopp.

Email Id: mahvashiram@gmail.com 
These ARBs have a specific ring structure (tetrazole) whose synthesis could potentially lead to the formation of nitrosamine impurities. Nirosamines can form during the production of $A R B s$ when certain reactions take place. The FDA had published the method for testing ARBs for nitrosamine impurities in the APIs, such as valsartan, which have been the subject of recalls and investigation dating back to 2018 .

The FDA has consistently downplayed the actual risk to patients from the contamination, however, citing analyses that showed minimal if any increase in cancer risk. Nevertheless, new ARB recalls continued into 2019.

A latest drug molecule which is being observed with utmost seriousness for patient safety by FDA for the high levels of NDMA is ranitidine. Although no recall was ordered, the FDA said patients using OTC ranitidine "could consider using other OTC medicines approved for their condition". The agency has asked manufacturers of ranitidine to conduct their own laboratory testing to assess levels of NDMA in their ranitidine formulations and to send samples of these formulations to the FDA to be tested by its scientists.

In its update issued October 2, 2019, the FDA stated that the use of higher temperatures in the testing method adapted by a third-party laboratory generated very high levels of NDMA from ranitidine formulations. To date, the agency's early, limited testing has found unacceptable levels of NDMA in samples of ranitidine. In announcing the ranitidine contamination, the FDA said it was still evaluating the level of risk to patients. It did not indicate whether the same was a class effect with all $\mathrm{H}_{2}$ blockers.

Patients and health care professionals should report any adverse event (mild/serious) to the nearest ADR monitoring centre at earliest. You can be a huge contributor for ensuring safety of millions.

Stay healthy!! 